\title{
Hibridación de modelos pedagógicos en la práctica docente en la educación superior en Ecuador. Caso Universidad Técnica de Ambato
}

\author{
Hybridization of pedagogical models in teaching practice in higher \\ education in Ecuador. Technical University of Ambato - Case study
}
Hibridação de modelos pedagógicos na prática docente no ensino superior no Equador. Caso Universidade Técnica de Ambato

ISSN 1688-9304 - DOI: https://doi.org/10.18861/cied.2020.11.1.2944

Luis Marcelo Mantilla-Falcón*1

https://orcid.org/0000-0002-8209-7365

Darwin Patricio Miranda Ramos ${ }^{\star 2}$

https://orcid.org/0000-0001-5611-9535

Grace Elaine Ortega Zurita ${ }^{* * * 3}$

https://orcid.org/0000-0002-0536-6672

Carlos Fernando Meléndez-Tamayo ${ }^{* \star *} 4$

https://orcid.org/0000-0002-7990-4859

Fecha de recibido: 23/05/2019

Fecha de aceptado: 27/11/2019

\section{Resumen}

En la educación superior latinoamericana, y muy particularmente en la ecuatoriana, los modelos pedagógicos en la práctica educativa se evidencian disímiles y dispares. La mayoría del personal docente no necesariamente pasó por las aulas de pedagogía y didáctica y, en consecuencia, se hizo docente al andar. Sin embargo, a la hora de su ejercicio profesional pone en práctica algunos modelos, ya sea de manera consciente o inconsciente, para ejecutar su labor docente. Se entiende como modelo pedagógico al compendio y abstracción de diversas teorías o enfoques que encaminan a los docentes hacia la preparación de los programas de estudio y a la dinámica misma de la praxis educativa. Esta es una investigación de carácter descriptivo, transversal, no experimental con muestreo no probabilístico y básicamente cuantitativo. El test utilizado en la recolección de datos cuenta con 40 reactivos cuyo alfa de Cronbach es de 0,911 y pertenece a De Zubiría (Fundación Universitaria Luis Amigó, 2009). Se analizó la presencia de cuatro enfoques-modelos pedagógicos de mayor recurrencia: el tradicionalista, el conductivo, el desarrollista y el social en correspondencia con el género, la experiencia docente, la facultad de pertenencia laboral y la edad de los docentes como criterios o variables implicados. Se realizó un análisis descriptivo a nivel porcentual de sus variables y, asimismo, se verificó la correlación interna entre modelos. Los principales resultados demuestran una alta correlación y la evidencia incuestionable de la hibridación de modelos pedagógicos en la práctica docente en la universidad.

Palabras Clave: Aprendizaje, docente universitario, educación superior, hibridación pedagógica, modelo pedagógico. 


\section{Abstract}

Pedagogical models in Higher Education in Latin America, and particularly in Ecuador, are dissimilar as regards educational practices. The majority of the teaching staff has not necessarily attended pedagogy and didactics courses, and consequently, most of them have become teachers as they went. However, at the moment of putting into practice their professional knowledge as they teach, they will be applying some models, either consciously or unconsciously. A pedagogical model is understood as the compendium and abstraction of several theories or approaches that guide teachers in the preparation of classes and programs as well as the dynamics of educational praxis itself. The present study is a descriptive, transversal, non-experimental research with non-probabilistic sampling, and basically a quantitative one. The test used for data collection has 40 items with a Cronbach's alpha of 0.911, and belongs to De Zubiria (Fundacion Universitaria Luis Amigo, 2009.) The following four approaches-pedagogical models of highest recurrence have been studied, to wit: traditionalist, conductive, developmental and social, according to gender, teaching experience, faculty where the teacher works, and age, as the criteria/ variables involved. A descriptive analysis of the variables' percentage level has been carried out and, in addition, the internal correlation between models has been verified. The main results show a high correlation and the unquestionable evidence of the crossbreeding of pedagogical models in the teaching practice at university.

Keywords: Learning, university professor, higher education, pedagogical hybridization, pedagogical model.

\section{Resumo}

No ensino superior latino-americano, e particularmente no Equador, a presença de modelos pedagógicos na prática educacional se reflete de maneira diversa e díspar. A maioria do corpo docente não passou necessariamente por aulas de pedagogia e didática; consequentemente, tornou-se professor ao caminhar. No entanto, em ocasião de sua prática profissional, ele pratica alguns modelos, consciente ou inconscientemente, para realizar seu trabalho de ensino. O modelo pedagógico é entendido como compêndio e abstração de várias teorias ou abordagens que orientam os professores na preparação de programas dos cursos e na dinâmica da práxis educacional. Trata-se de uma pesquisa descritiva, transversal, não experimental, com amostragem não probabilística e basicamente quantitativa. O teste utilizado na coleta de dados possui 40 reagentes cujo alfa de Cronbach é 0,911 e pertence a De Zubiría (Fundação Universidade Luis Amigó, 2009). Foi analisada a presença de quatro abordagens-modelo pedagógicas de maior recorrência: tradicionalistas, condutores, desenvolvimentistas e sociais em correspondência com gênero, experiência docente, pertença à Faculdade Trabalhista e idade como critério ou variável envolvida. Foi realizada uma análise descritiva no nível percentual de suas variáveis e, além disso, foi verificada a correlação interna entre os modelos. Os principais resultados mostram uma alta correlação e evidências indiscutíveis de hibridização de modelos pedagógicos na prática docente na universidade.

Palavras chave: Aprendizagem, professor universitário, ensino superior, hibridação pedagógica, modelo pedagógico. 


\section{Introducción}

El espectro del profesor universitario no consiste necesariamente en profesionales con formación y verdadera vocación docente. Por el contrario, son varios los factores que llevan a involucrarse en la docencia universitaria puesto que la gama de profesionales va desde las ciencias humanas, técnicas, naturales y médicas hasta las filosóficas, y vincular el conocimiento científico del área específica con la pedagogía y didáctica tiene su complejidad. Este panorama condiciona a que el docente no tenga definida una identidad pedagógica en su práctica profesional y que maneje una hibridación importante entre los diferentes enfoques-modelos establecidos hasta la actualidad.

La complejidad del hecho educativo es significativa y no pasa solo por la aplicación de enfoques y modelos pedagógicos sino, como bien lo expresa Díaz-Quero (2006),

"Ios escenarios donde se desarrolla la actividad docente, escuela, colegio, universidad, son entidades complejas, dinámicas y dialécticas que tienen como propósitos centrales: (a) transmitir y mantener los valores de la cultura de una sociedad a través de un currículo, (b) promover los cambios socio-culturales de su entorno y (c) contribuir con la formación personal y profesional de la población" (p. 89).

Barajas (2013), citando a Morales (2000) clarifica los conceptos relativos a este estudio para su cabal entendimiento en el marco del fenómeno educativo universitario. "Un enfoque es una manera de concebir, organizar y realizar la educación y el aprendizaje, que puede dar origen y fundamento a distintas corrientes y modelos pedagógicos" (p. 13).

Evidentemente, un gran corpus de profesionales trabaja en las teorías que sirven de base para la construcción de los enfoques pedagógicos, ya se trate de sicólogos, sociólogos o investigadores educativos de gran nombre, y bajo sus premisas y postulados se hacen interpretaciones y se formulan criterios, lineamientos, políticas y estrategias pedagógicas (Barajas, 2013, p. 13).

Consolidando el criterio del constructo enfoques que por principio son "concepciones y percepciones abiertas, flexibles e hipotéticas de la realidad educativa, que permiten ajustes, adaptaciones e integraciones de las distintas ciencias y de diferentes experiencias pedagógicas, conservando siempre una perspectiva idiosincrática en el análisis del fenómeno educativo" (Barajas, 2013, p. 13) se entiende la verdadera complejidad de la praxis en la dinámica del hecho educativo.

Para muchos, el concepto de modelo pedagógico se mimetiza con el de enfoque pedagógico, aunque son dos términos muy diferentes. Un modelo pedagógico define un conjunto de atributos que caracterizan el proceso de la educación y formación (Pinilla, 2011, p. 205), “que se construye y orienta según un método históricamente determinado por una concepción del hombre, la sociedad y el conocimiento" (Claret, 2003, p. 16).

Dada la confusión en cuanto a la identificación clara y concreta de cuál es su modelo pedagógico de enseñanza, para los docentes se difuminan en un mar de apreciaciones y divagaciones sin certezas y sin referentes precisos de su práctica pedagógica y en muchos casos se ven abocados a reproducir los modelos de sus antiguos profesores y a "copiar" detalles particulares. 
En términos de Pineda-Báez, Hennig y Segovia (2013)

"Algunos docentes/tutores reconocen que, aunque las instituciones cuentan con modelos pedagógicos, estos se tornan ambiguos, resultan insuficientes para guiar a los profesores en su labor pedagógica y se conciben como ideales o utópicos. Al parecer, la ambigüedad en el manejo de términos proviene de vacíos conceptuales y de una insuficiencia en la socialización de los modelos, y de las estrategias y herramientas para ponerlos en marcha" (p. 192).

Los criterios de los académicos en función de la realidad pedagógica de la educación superior ecuatoriana se resumen en las siguientes palabras:

“Los modelos pedagógicos que predominan en la planificación y operatividad de la práctica docente universitaria son de tendencia tradicional y conductista, desvinculados de la problemática de la realidad social, que al objetivarse fundamentalmente en el aula se constituyen en obstáculos epistemológicos, ontológicos y metodológicos para integrar las funciones sustantivas de la universidad e intervenir productivamente en el desarrollo social" (Cartuche, Tusa, Aguinsaca, Merino \& Tene, 2015, p. 229).

La trasmisión del saber pedagógico se corresponde con el paradigma al que pertenece. En la dinámica educativa, la identificación del docente con un modelo pedagógico determinado se refleja en la forma en la que este define y despliega la acción educativa. Entonces es evidente que la caracterización y categorización de la forma de aplicación de los saberes en mención son un pilar fundamental de los conocimientos tanto científicos como pedagógicos (Almonacid-Fierro, Merellano-Navarro \& Moreno-Doña, 2014).

La literatura evidencia que las instituciones educativas promueven estrategias de capacitación orientadas a la difusión de procesos educativos de interaprendizaje, mas en la práctica es muy común que el hecho educativo se finque en el paradigma tradicional, así como también que se determine la aplicación del modelo conductista como esencial (Almonacid-Fierro, Merellano-Navarro \& Moreno-Doña, 2014).

Es necesario mencionar que, en la educación superior, los modelos pedagógicos siempre han estado en permanente cambio. Sin embargo, es el tradicional el que ha evolucionado desde el mundo griego y que resulta predominante en la cultura occidental (De Jesús, Méndez, Andrade \& Martínez, 2007).

Concomitante con el estudio es significativo señalar que la universidad ha comulgado estrictamente con los principios del tradicionalismo, haciendo de los profesores entes con la capacidad exclusiva de transmitir, traspasar y transferir un cúmulo de conocimientos, informaciones y experiencias (De Jesús, Méndez, Andrade \& Martínez, 2007, p. 19). Este es un problema que aún subsiste en la práctica pedagógica. Llevará mucho tiempo aun romper con los paradigmas de largo alcance que tienen una profunda presencia en el quehacer educativo latinoamericano.

Flórez-Ochoa (2005, p. 159) define a los modelos pedagógicos como "categorías descriptivoexplicativas, auxiliares para la estructuración teórica de la pedagogía, pero solo adquieren sentido al contextualizarlos históricamente". Es necesario que se enfatice tanto el trabajo del sujeto de aprendizaje como el del docente para aplicarlos de la manera más adecuada y eficiente en la formación del estudiante. 
La actividad diaria que se desarrolla en las aulas, laboratorios u otros espacios orientada por un currículo y que tiene como propósito la formación de los alumnos es la práctica pedagógica (Díaz-Quero, 2006, p. 90) y, son precisamente el momento, el espacio y las circunstancias donde el docente visibiliza su potencial académico apoyado por una praxis pedagógica gracias a la cual se convierte en enseñador, transmisor, formador o mediador de los conocimientos según su estilo y el modelo que practique.

En este estudio se caracterizan y consideran cuatro modelos en discusión: el tradicionalista, el conductista, el social y el desarrollista, cada uno con sus particularidades y similitudes si las hubiere, pero que están presentes en el hecho educativo.

Caracterizando la práctica pedagógica de acuerdo con el modelo-enfoque de enseñanza, el docente tradicionalista en su forma más clásica "enfatiza en la formación del carácter de los estudiantes para moldear, a través de la voluntad, la virtud y el rigor de la disciplina, el ideal humanístico y ético que recoge la tradición metafísico-religiosa medieval" (FlórezOchoa, 1999, p. 33).

El docente que acoge el modelo social como su timón de práctica docente prioriza el trabajo en grupos para la atención de problemas que se corresponden con la realidad concreta de los estudiantes, sin apartarse de su contexto social. En este modelo se requiere de un educador capaz de cuestionarse, abrirse al cambio y vivenciar coherentemente su discurso. La dinámica pedagógica "se realiza en un marco de tolerancia, diálogo real y respeto a las ideas de los demás" (Castillo-Cedeño, Flores-Davis, Jiménez-Corrales \& Perearnau-Torras, 2008, p. 127).

Bajo la práctica del conductismo el docente básicamente busca "la fijación y control de los objetivos instruccionales formulados con precisión y reforzados minuciosamente. Adquirir conocimientos, códigos impersonales, destrezas y competencias bajo la forma de conductas observables, es equivalente al desarrollo intelectual del estudiante" (MoralesGómez, 2004, p. 107).

El modelo desarrollista, también conocido como cognoscitivista, tiene como meta educativa "que cada individuo acceda, progresiva y secuencialmente a la etapa de desarrollo intelectual, de acuerdo con las necesidades y condiciones de cada uno" (Flórez-Ochoa, 1994, p. 169).

“En el modelo cognoscitivista el rol del maestro está dirigido a tener en cuenta el nivel de desarrollo y el proceso cognitivo de los alumnos. El maestro debe orientar a los estudiantes a desarrollar aprendizajes por recepción significativa y a participar en actividades exploratorias, que puedan ser usadas posteriormente en formas de pensar independiente" (Pinto-Blanco \& Castro-Quitora, 2008, p. 6).

Este trabajo de investigación describe las prácticas pedagógicas de los docentes de la Universidad Técnica de Ambato, Ecuador, en sus diferentes situaciones de enseñanzaaprendizaje. En la revisión teórica se evidencia la conceptualización y diferenciación entre los enfoques tradicional, conductista, desarrollista (cognitivismo) y social (constructivismo). Dicho de otra manera, "Estas teorías son un marco de referencia para vislumbrar los principios pedagógicos que favorezcan el aprendizaje significativo de los estudiantes y cómo los docentes asumen el rol de facilitadores en el proceso de formación" (González Guerrero \& Esteban Ojeda, 2013, p. 5). 
El propósito del estudio consiste en evidenciar que no hay modelos puros ni exclusivos en la práctica pedagógica sino que se genera una hibridación notoria que va evolucionando conforme avanzan la experiencia y la acción docente.

\section{Metodología}

Se trata de una investigación cuantitativa de carácter descriptivo, transversal, no probabilístico y no experimental (Hernández, Fernández \& Baptista, 2016). Los elementos de observación fueron 221 docentes de la Universidad Técnica de Ambato, UTA, Ecuador; $30,3 \%$ a nombramiento y $69.7 \%$ de contrato, pertenecientes a diez facultades. De dicho número el 34,4\% pertenecía al género femenino y $65,6 \%$ al masculino. La recolección de la información fue realizada mediante plataforma virtual aprovechando la coyuntura de un curso intersemestral en el que participaron 650 integrantes. Los mismos fueron invitados a completar la encuesta Identificación de enfoques-modelos pedagógicos adaptada de Julián de Zubiría (Fundación Universitaria Luis Amigó, 2009) que permite identificar el enfoque-modelo pedagógico de la práctica docente. La encuesta consta de 40 ítems, con 10 preguntas en distinto orden para determinar los modelos: tradicionalista, conductista, desarrollista y social. El instrumento está formulado con escala de Likert para la que $0=$ nada, $1=$ un poco, 2=bastante y 3=totalmente. Se realizó una jerarquización de los enfoques-modelos bajo las siguientes consideraciones: 0=nada; de 1 a 10=un poco; de 11 a 20 puntos=bastante y de 21 a 31 puntos=totalmente. La fiabilidad para alfa (a) de Cronbach (Quero Virla, 2010) del test completo fue de 0,911 y por modelos es como sigue: tradicionalista, 0,793; conductista, 0,773; desarrollista, 0,745; y social, 0,721.

\section{Resultados}

La caracterización de los resultados empieza por una descripción y sistematización de cómo se encuentra distribuida la muestra donde figuran las diez facultades con las que cuenta la universidad más el Departamento de idiomas.

\section{Tabla 1. Caracterización de la muestra}

\begin{tabular}{|c|l|c|c|c|c|c|c|}
\hline $\mathbf{N}^{\circ}$ & \multicolumn{1}{|c|}{ Facultades } & \multicolumn{3}{c|}{ Género } & \multicolumn{3}{c|}{ Relación de dependencia } \\
\hline & & M. & F. & Total & Nombr. & Contr. & Total \\
\hline $\mathbf{1}$ & $\begin{array}{l}\text { Ciencia en Ingeniería en } \\
\text { Alimentos }\end{array}$ & 19 & 5 & 24 & 2 & 22 & 24 \\
\hline $\mathbf{2}$ & $\begin{array}{l}\text { Ciencias Humanas y de la } \\
\text { Educación }\end{array}$ & 21 & 25 & 46 & 13 & 33 & 46 \\
\hline $\mathbf{3}$ & Ciencias de la Salud & 10 & 17 & 27 & 1 & 26 & 27 \\
\hline $\mathbf{4}$ & Ciencias Administrativas & 22 & 5 & 27 & 10 & 17 & 27 \\
\hline $\mathbf{5}$ & Ciencias Agropecuarias & 9 & 3 & 12 & 7 & 5 & 12 \\
\hline $\mathbf{6}$ & Diseño arquitectura y artes & 2 & 1 & 3 & 0 & 3 & 3 \\
\hline $\mathbf{7}$ & Ingeniería Civil y Mecánica & 12 & 2 & 14 & 5 & 9 & 14 \\
\hline $\mathbf{8}$ & $\begin{array}{l}\text { Ingeniería en Sistemas, } \\
\text { Electrónica e Industrial }\end{array}$ & 19 & 3 & 22 & 7 & 15 & 22 \\
\hline $\mathbf{9}$ & $\begin{array}{l}\text { Jurisprudencia y Ciencias } \\
\text { Sociales }\end{array}$ & 7 & 0 & 7 & 4 & 3 & 7 \\
\hline $\mathbf{1 0}$ & Contabilidad y Auditoría & 24 & 14 & 38 & 17 & 21 & 38 \\
\hline $\mathbf{1 1}$ & Centro de Idiomas & 0 & 1 & 1 & 1 & 0 & 1 \\
\hline & Total & 145 & 76 & 221 & 67 & 154 & 221 \\
\hline
\end{tabular}

Fuente: Elaboración propia 
Una descripción rápida de la composición general del plantel docente en la UTA deja ver la realidad tanto en lo referente a género como a relación de dependencia, lo que es motivo de análisis sociológico y antropológico. La mayoría de las carreras técnicas tienen supremacía de docentes varones, excepto la facultad de Ciencias Humanas y de la Educación en la que se forman profesionales parvularios, psicólogos educativos, docentes de educación básica y cuya población estudiantil es mayormente femenina (Mantilla-Falcón, Galarza-Galarza \& Zamora-Sánchez, 2017). Este sesgo se comprende fácilmente en base a la creencia de que "las acciones docentes y administrativas en la educación superior están siendo permeadas por constructos sociales dentro de los cuales cobran particular importancia aquellas que establecen diferencias de comportamiento asignado a hombres y a mujeres en los diversos escenarios de interacción humana, específicamente en la educación superior, llegando a clasificar por género hasta la oferta académica de las instituciones" (Restrepo-García, Franco-Muñoz \& Quiroz-Posada, 2011).

Tal como se evidencia en el presente estudio y hasta la fecha del mismo (marzo-2016), desde el punto de vista laboral la universidad contaba con un número mayor de docentes a contrato (70\% aproximadamente) y con un muy reducido el número de profesores con nombramiento (30\%). Las condiciones de estabilidad laboral y la relación de dependencia están ligadas a factores económicos de presupuesto y a la voluntad política nacional e institucional.

La UTA es una Institución de Educación Superior-IES con más de 50 años de existencia y que cuenta en su haber con 800 docentes, 16.000 estudiantes y algo más de 500 empleados administrativos y de servicio. Cuenta con diez facultades, 40 carreras de pregrado y varios programas de posgrado.

De acuerdo con el test aplicado y en función de los baremos diseñados a tales efectos al cruzar la información recogida los resultados se configuran como se detalla en la tabla 2. Es necesario aclarar que el test trae señaladas qué preguntas corresponden a cada enfoquemodelo pero ese detalle no es de conocimiento del encuestado, solo del investigador con el fin de evitar direccionamientos o sesgos en las respuestas.

\section{Tabla 2. Distribución de los enfoques-modelos de enseñanza}

\begin{tabular}{|c|c|c|c|c|c|c|c|c|}
\hline VALORACIÓN & \multicolumn{6}{|c|}{ ENFOQUES-MODELOS PEDAGÓGICOS DE ENSENANZA } \\
\hline & \multicolumn{2}{|c|}{ Tradicional } & \multicolumn{2}{c|}{ Conductista } & \multicolumn{2}{c|}{ Desarrollista } & \multicolumn{2}{c|}{ Social } \\
\hline & $\mathrm{f}$ & $\%$ & $\mathrm{f}$ & $\%$ & $\mathrm{f}$ & $\%$ & $\mathrm{f}$ & $\%$ \\
\hline Un poco & 14 & 6,3 & 3 & 1,4 & 2 & 0,9 & 0 & 0,0 \\
\hline Bastante & 151 & 68,3 & 101 & 45,7 & 142 & 64,3 & 92 & 41,6 \\
\hline Totalmente & 56 & 25,3 & 117 & 52,9 & 77 & 34,8 & 129 & 58,4 \\
\hline TOTAL & 221 & 100 & 221 & 100 & 221 & 100 & 221 & 100 \\
\hline
\end{tabular}

Fuente: Elaboración propia

Para comprender los resultados de la práctica de los enfoques-modelos de enseñanza debe aclararse que todos los docentes evidencian la existencia de los cuatro componentes. Sin embargo, uno de estos es el más visible y el de mayor recurrencia en la práctica pedagógica o, como sucede en algunos casos, son dos los más presentes. La cartografía de la realidad educativa en cuanto a la práctica pedagógica del docente universitario evidencia que más del $25 \%$ sigue siendo totalmente tradicionalista. Le sigue en orden ascendente el modelo desarrollista con el 35\% aproximadamente; el siguiente lugar lo ocuparía el modelo 
conductista con el 52,9\% y finalmente con el 58,4\% el modelo social, considerando el modelo como el $100 \%$ de análisis. En el conteo de las respuestas en forma independiente por modelo (884), considerando la valoración de su accionar en la práctica docente como el $100 \%$, los datos demuestran la hibridación existente en la labor pedagógica tal como se verifica en la tabla 3.

La secuencia descendente de los modelos pedagógicos en la práctica docente es como sigue: el 34,04\% se declara totalmente social, el 30,87\% conductista, el 20,32\% desarrollista y el $14,78 \%$ tradicionalista.

Como ningún docente tiene exclusividad absoluta en cuanto a un modelo determinado sino que, por el contrario, también evidencian características de los modelos complementarios, el estudio demuestra que el 31,07\% es bastante tradicionalista, el 29,22\% es desarrollista, el $20,78 \%$ es conductista y el $18,93 \%$ se considera social, quedando así demostrada la verdadera hibridación de modelos pedagógicos con una clara complementariedad u oposición en el orden de importancia y recurrencia.

Tabla 3. Relación entre modelos y recurrencia de aplicación

\begin{tabular}{|l|l|l|l|l|l|}
\hline & \multicolumn{4}{|c|}{ ENFOQUES-MODELOS } & Total \\
\hline Valoración & Tradicional & Conductista & Desarrollista & Social & \\
\hline & $\mathrm{f}(\%)$ & $\mathrm{f}(\%)$ & $\mathrm{f}(\%)$ & $\mathrm{f}(\%)$ & $\mathrm{f}(\%)$ \\
\hline Un poco & $14(73,7 \%)$ & $3(15,8 \%)$ & $2(10,5 \%)$ & $0(0,00 \%)$ & $19(100 \%)$ \\
\hline Bastante & $151(31,1 \%)$ & $101(20,8 \%)$ & $142(29,2 \%)$ & $92(18,9 \%)$ & $486(100 \%)$ \\
\hline Totalmente & $56(14,8 \%)$ & $117(30,9 \%)$ & $77(20,3 \%)$ & $129(34,0 \%)$ & $379(100 \%)$ \\
\hline
\end{tabular}

Fuente: Elaboración propia

La contrastación de la información con un análisis de Ji cuadrado arroja los siguientes resultados: $\mathrm{X}_{(83,02)} ; p=0,0000$ lo que significa que hay diferencia estadística significativa entre modelos e importancia de su recurrencia.

Para la comprensión de cómo se distribuyen los modelos-enfoques por cada facultad de la universidad los datos son más que evidentes, tal como se detalla en la tabla 4. 
Hibridación de modelos pedagógicos en la práctica docente en la educación superior en Ecuador...

Tabla 4. Distribución de los enfoques-modelos de enseñanza por facultades

\begin{tabular}{|l|c|c|c|c|c|c|c|c|}
\hline \multicolumn{1}{|c|}{ FACULTADES } & \multicolumn{6}{|c|}{ ENFOQUES-MODELOS PEDAGÓGICOS DE ENSEÑANZA } \\
\hline & \multicolumn{2}{|c|}{ Tradicional } & \multicolumn{2}{c|}{ Conductista } & \multicolumn{2}{c|}{ Desarrollista } & \multicolumn{3}{c|}{ Social } \\
\hline & $\mathrm{f}$ & $\%$ & $\mathrm{f}$ & $\%$ & $\mathrm{f}$ & $\%$ & $\mathrm{f}$ & $\%$ \\
\hline Ing. Alimentos & 6 & 10,7 & 11 & 9,4 & 8 & 10,4 & 12 & 9,3 \\
\hline $\begin{array}{l}\text { Ciencia Humana } \\
\text { y Educación }\end{array}$ & 14 & 25,0 & 21 & 17,9 & 22 & 28,6 & 36 & 27,9 \\
\hline Salud & 6 & 10,7 & 12 & 10,3 & 8 & 10,4 & 14 & 10,9 \\
\hline Administración & 6 & 10,7 & 22 & 18,8 & 12 & 15,6 & 18 & 14,0 \\
\hline $\begin{array}{l}\text { Ing. Ciencias } \\
\text { Agropecuarias }\end{array}$ & 4 & 7,1 & 7 & 6,0 & 4 & 5,2 & 8 & 6,2 \\
\hline Arquitec. Artes & 2 & 3,6 & 2 & 1,7 & 2 & 2,6 & 2 & 1,6 \\
\hline $\begin{array}{l}\text { Ingeniería Civil- } \\
\text { Mecánica }\end{array}$ & 3 & 5,4 & 7 & 6,0 & 4 & 5,2 & 9 & 7,0 \\
\hline $\begin{array}{l}\text { Ing. Sistem. } \\
\text { Electrónica }\end{array}$ & 5 & 8,9 & 12 & 10,3 & 7 & 9,1 & 12 & 9,3 \\
\hline $\begin{array}{l}\text { Juris-Ciencias- } \\
\text { Sociales }\end{array}$ & 1 & 1,8 & 4 & 3,4 & 2 & 2,6 & 5 & 3,9 \\
\hline $\begin{array}{l}\text { Contabilidad- } \\
\text { Auditoría }\end{array}$ & 9 & 16,1 & 19 & 16,2 & 8 & 10,4 & 12 & 9,3 \\
\hline $\begin{array}{l}\text { Centro de } \\
\text { Idiomas }\end{array}$ & 0 & 0,0 & 0 & 0,0 & 0 & 0,0 & 1 & 0,8 \\
\hline \begin{tabular}{l} 
TotAL \\
\hline
\end{tabular} & 56 & 100 & 117 & 100 & 77 & 100 & 129 & 100 \\
\hline
\end{tabular}

Fuente: Elaboración propia

La información evidencia que, en concordancia con el enfoque de la práctica pedagógica -en este caso, el tradicionalista- la Facultad de Ciencias Humanas y de la Educación es la que mayor porcentaje representa (25\%), seguida por la Facultad de Contabilidad y Auditoría. Tomando en consideración el modelo conductista la Facultad de Administración y Ciencias Humanas y de la Educación lideran esta práctica pedagógica. Nuevamente la Facultad de Ciencias Humanas y de la Educación evidencia el más alto porcentaje en cuanto a la práctica del modelo desarrollista $(28,6 \%)$ y, en menor porcentaje, el social $(27,9 \%)$, analizadas en función del modelo pedagógico.

Con un análisis más detallado y profundo la Facultad de Ciencias Humanas y de la Educación lidera con la presencia de los 4 enfoques-modelos de enseñanza. El más evidente es el social con el 9,5\%, seguido del desarrollista con el 5,8\%, el conductista con el 5,5\% y finalmente el tradicionalista con el 3,7\%. Significa entonces que hay clara presencia de los cuatro estilos de enseñanza arraigados en los docentes con los que se forma y que se transmite a los dicentes o futuros maestros y formadores de generaciones venideras. Esta gama de prácticas pedagógicas es lo que se denomina hibridación pedagógica docente, donde se entrecruzan y amalgaman los diferentes estilos de enseñanza que le dan más riqueza y potencialidad al hecho educativo universitario.

El panorama general del hibridismo pedagógico docente en la UTA se estructura de manera global con los siguientes referentes: el 34\%, social; el 31\%, conductista; el 20\%, desarrollista y $15 \%$ tradicionalista. 
Para comprender la fenomenología de la práctica docente y la evolución de los modelos a través de los años de experiencia, en la tabla 5 se presenta esta información.

Tabla 5. Los enfoques-modelos de enseñanza por años de experiencia

\begin{tabular}{|l|l|l|l|l|l|l|l|l|}
\hline $\begin{array}{c}\text { AÑOS DE } \\
\text { EXPERIENCIA }\end{array}$ & \multicolumn{6}{c|}{ ENFOQUES-MODELOS PEDAGÓGICOS DE ENSEÑANZA } \\
\hline & \multicolumn{2}{|c|}{ Tradicional } & \multicolumn{2}{|c|}{ Conductista } & \multicolumn{2}{c|}{ Desarrollista } & \multicolumn{2}{c|}{ Social } \\
\hline & $\mathrm{f}$ & $\%$ & $\mathrm{f}$ & $\%$ & $\mathrm{f}$ & $\%$ & $\mathrm{f}$ & $\%$ \\
\hline de 1 a $\mathbf{1 0}$ & 45 & 80,4 & 81 & 69,2 & 41 & 53,2 & 68 & 52,7 \\
\hline de 11 a $\mathbf{2 0}$ & 10 & 17,9 & 29 & 24,8 & 28 & 36,4 & 48 & 37,2 \\
\hline de $\mathbf{2 1}$ a $\mathbf{3 0}$ & 1 & 1,8 & 7 & 6,0 & 8 & 10,4 & 13 & 10,1 \\
\hline TOTAL & 56 & 100 & 117 & 100 & 77 & 100 & 129 & 100 \\
\hline
\end{tabular}

Fuente: Elaboración propia

Los datos demuestran que en el rango de los primeros 10 años de práctica docente se acumulan los mayores porcentajes de presencia de los cuatro estilos de enseñanza siendo el modelo conductista el de mayor significación. En un análisis global en función de la experiencia docente (cruce de los 4 modelos por años de experiencia: tabla de contingencias) se ratifica que el modelo conductista es el que domina el escenario pedagógico en la Universidad Técnica de Ambato con el 21,4\% de presencia y corresponde a los docentes con experiencia inferior a los diez años. Le sigue el modelo social con el $17,9 \%$, luego el tradicional con el 11,9\% y finalmente el desarrollista con el 10,8\%. Entre los 11 y los 20 años y entre los 21 y los 30 años de experiencia el modelo desarrollista figura entre los primeros.

Por medio de la aplicación de una prueba estadística se determina que hay diferencia estadística significativa entre los años de experiencia y la práctica de los modelos aplicados en la labor docente. La prueba del Ji cuadrado de Fisher arroja el siguiente indicador: $\mathrm{X}^{2}$ $(18,46) ; p=0,0052$.

La práctica pedagógica en función del género del docente brinda información relevante. El modelo desarrollista lidera con el $67,5 \%$ en los varones y el modelo conductista llega al $34,2 \%$ en las mujeres (ver gráfico 1). 




Gráfico 1. Porcentajes de correspondencia entre los enfoques-modelos pedagógicos y el género del docente

De manera global en la UTA, tanto varones como mujeres aplican el modelo social, que se ubica en los primeros puestos: $22,7 \%$ y $11,3 \%$ respectivamente. La variación radica en el modelo que ocupa el último lugar: el 9,8\% de varones y el 5,0\% de mujeres son tradicionalistas (ver tabla 6), pero con un análisis global la prueba de Ji cuadrado determina que no hay diferencia estadística significativa $\left(X_{(8,47)}^{2} ; p=0,2054\right)$.

Tabla 6. Porcentaje de recurrencia de aplicación del enfoque-modelo por género

\begin{tabular}{|c|c|c|c|c|c|}
\hline GÉNERO & \multicolumn{3}{|c|}{ ENFOQUES-MODELOS PEDAGÓGICOS DE ENSEÑANZA } & TOTAL \\
\hline & \% Tradicional & \% Conductista & $\begin{array}{c}\% \\
\text { Desarrollista }\end{array}$ & \% Social & \\
\hline Masculino & 9,8 & 20,3 & 13,7 & 22,7 & 66,5 \\
\hline Femenino & 5,0 & 10,6 & 6,6 & 11,3 & 33,5 \\
\hline TOTAL & 14,8 & 30,9 & 20,3 & 34,0 & 100,0 \\
\hline
\end{tabular}

Fuente: Elaboración propia

Una particularidad especial y muy importante en la práctica docente es la edad del maestro. Los siguientes datos reflejan esa realidad en este estudio. 


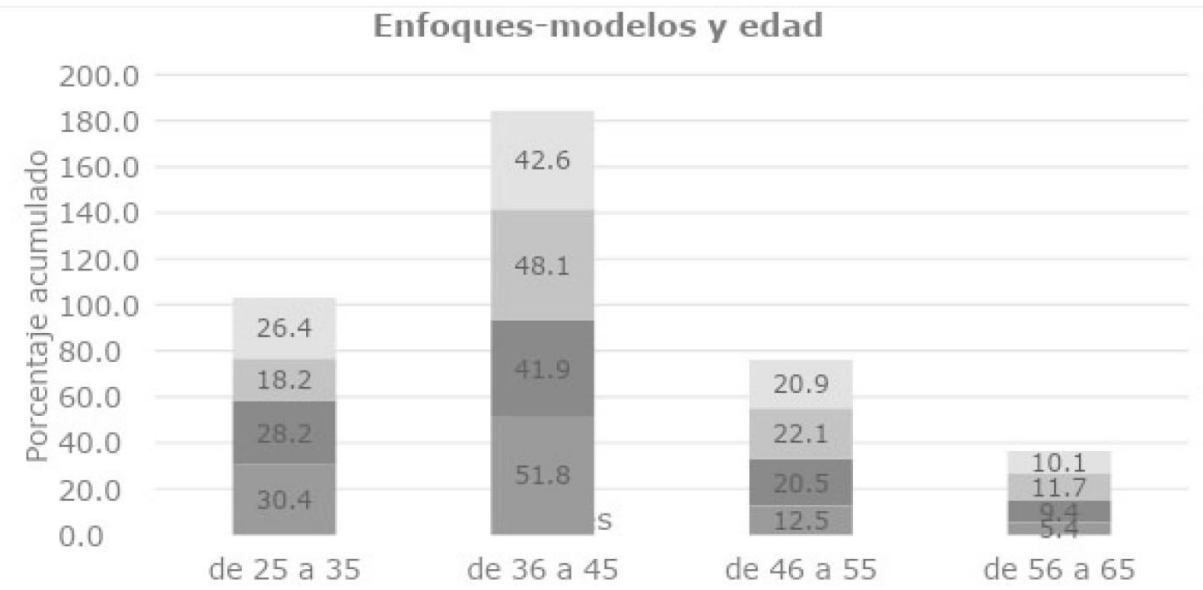

Tradicional a Conductista Desarrollista Social

\section{Gráfico 2. Porcentajes de recurrencia de los enfoques-modelos pedagógicos y edades de los docentes.}

Considerando la edad cronológica del docente, entre los 36 y los 45 años se evidencia el mayor porcentaje de presencia de los cuatro modelos de enseñanza, siendo el tradicional el que tienen mayor presencia: 51,8\%. Una consecuencia directa que va en forma paralela a los años de experiencia y a la edad del docente es la variación en el modelo de práctica pedagógica. Esto significa que solo el 5,4\% de los docentes de alrededor de 60 años de edad se considera tradicionalista.

Tabla 7. Correlación interna entre enfoques-modelos

\begin{tabular}{|l|c|c|c|c|}
\hline MODELOS & \multicolumn{2}{|c|}{ TRADICIONAL } & CONDUCTISTA & DESARROLLISTA \\
\hline CONDUCTISTA & $r$ &, $762^{* *}$ & & \\
\hline DESARROLLISTA & $r$ &, $623^{* *}$ &, $525^{* *}$ & \\
\hline SOCIAL & $r$ &, $549^{* *}$ &, $478^{* *}$ &, $687^{* *}$ \\
\hline \multirow{2}{*}{ **. La correlación es significativa al nivel 0,01 (bilateral). } \\
\hline
\end{tabular}

La información de mayor relevancia en el análisis correlacional se fundamenta en la estrecha vinculación entre los modelos tradicional y conductista cuyo $r$ de Pearson es de 0,762 lo que determina una correlación positiva fuerte, así como entre el desarrollista y social $(0,687)$, modelos que tienen origen en el constructivismo.

Establecida una asociación entre pares de modelos pedagógicos (tradicionalista vs. conductista) y la magnitud de ser totalmente, bastante y un poco de cada uno de los modelos, aplicado el estadígrafo del $\mathrm{X}^{2}$ (Ji cuadrado) los resultados son significativos en cuanto a las diferencias entre ellos y a la hibridación que representan. No hay prácticas pedagógicas exclusivas y puras sino la mezcla proporcional de ingredientes importantes de uno u otro modelo en la actividad educativa. Los resultados se evidencian en la siguiente tabla: 
Hibridación de modelos pedagógicos en la práctica docente en la educación superior en Ecuador...

Tabla 8. Contraste mediante Ji cuadrado

\begin{tabular}{|l|c|c|c|c|}
\hline Modelo 1 & Modelo 2 & X2 & gl & p-value \\
\hline Tradicionalista & Conductista & 103,502 & 4 & 0,0000 \\
\hline & Desarrollista & 45,106 & 4 & 0,0000 \\
\hline & Social & 24,205 & 2 & 0,0000 \\
\hline Conductista & Desarrollista & 22,875 & 4 & 0,0000 \\
\hline & Social & 23,514 & 2 & 0,0000 \\
\hline Desarrollista & Social & 47,518 & 2 & 0,0000 \\
\hline
\end{tabular}

Fuente: Elaboración propia

\section{Discusión}

La Universidad Técnica de Ambato, asumiendo el compromiso y responsabilidad social de proveer profesionales de alta calidad orientados al desarrollo local, regional y nacional y con una visión transformadora de la realidad, en su práctica pedagógica se apoya en los enfoques humanistas (social y desarrollista 54,36\%) que consideran la integralidad del individuo en su dimensión biopsicosocial como agente pensante, crítico, propositivo, dialéctico y dialógico capaz de entender el mundo desde la acción local. Se ratifica entonces una acción pedagógica cuyo "centro focal del acto del conocimiento, esto bajo el principio de la dialogicidad y mediado por la responsabilidad social, la pedagogía emancipatoria y la felicidad del ser humano en la construcción de la paz" (Soto Arango, Mora García \& Lima Jardilino, 2017, p. 36) así como en la preservación del medioambiente.

La práctica pedagógica cobra visibilidad debido a su problematización contemporánea, está presente en los discursos educativos recientes y pasa por lo económico y lo político. A partir de dicha práctica es posible intervenir modos individuales y colectivos de ser y estar en el mundo (Noguera-Ramírez \& Marín-Díaz, 2017). Esta complejidad se materializa por las múltiples relaciones que maneja: labor docente, cumplimiento de reglamentos y políticas, ajuste a un modelo pedagógico sugerido pero no necesariamente ejecutado, evaluaciones, requerimientos administrativos internos y observación de la política nacional, entre otras. La hibridación de los modelos se materializa dado que unos docentes llevan su accionar educativo centrado en el estudiante, característica particular y potencial de los modelos desarrollista, social y conductista, mientras otros lo hacen centrado en el docente, característica propia del modelo tradicionalista. La existencia de esta gama de modelos se infiere a la reproducción de los procesos de aprendizaje que recibieron cuando fueron estudiantes (Carrasco, Pérez, Torres \& Fasce, 2016) como así lo sugiere la bibliografía al respecto.

La distribución de modelos pedagógicos en la práctica docente de hombres y mujeres es semejante, lo que equivale a decir que el género no es determinante para aplicar un modelo pedagógico específico. Autores como Vaccarezza Garrido, Sánchez Soto y Alvarado Martínez (2018, p. 10) no encontraron diferencia significativa en la práctica pedagógica entre varones y mujeres en la carrera de ingeniería civil en las universidades de Chile. 


\section{Conclusiones}

En la docencia universitaria se evidencia claramente la presencia de los cuatro modelos analizados con un alto grado de participación. Esto corrobora la existencia de una visible hibridación en el accionar pedagógico en el cumplimiento del rol docente.

Las tendencias y puestas en práctica de los modelos pedagógicos no se diferencian entre hombres y mujeres, lo que significa que el género no es una categoría que incida en la aplicación de tal o cual modelo. Simplemente el docente lleva a cabo su actividad educativa en función de sus necesidades, saberes y hábitos pedagógicos.

Por las similitudes y elementos comunes de los modelos el conductista y el tradicionalista presentan una fuerte correlación $(r=0,762)$ evidenciándose así una notable hibridación en la práctica pedagógica. De igual forma los modelos desarrollista y social se ajustan plenamente entre ellos con un $r$ de 0,687. La correlación más baja se da entre los enfoques conductista y social pues sus prácticas, características y elementos son diametralmente opuestos.

La Universidad Técnica de Ambato evidencia una práctica pedagógica caracterizada, de mayor a menor, por los siguientes modelos: totalmente social $(34,04 \%)$, conductista $(30,87 \%)$, desarrollista $(20,32 \%)$ y tradicionalista $(14,78 \%)$.

Según García Jiménez (2009, p. 8), en la realidad educativa en todos los niveles al parecer existen dos tipos de docentes: los que quieren transformar el centro educativo y, por tanto, están conscientes de su rol dentro de la sociedad y buscan su proyección social y trascendencia, y aquellos que ven la realidad y la dejan tal cual y solo se dedican a cumplir mínimos establecidos sin comprometerse a modificar nada de lo existente.

La literatura científica asevera que "no existe un único modelo que sea adecuado para cualquier contexto educativo, por lo que se plantea la hibridación o combinación de partes de varios de ellos para desarrollar todo su potencial y adaptarse a las necesidades de los estudiantes" (Antón-Candanedo \& Fernández-Río, 2017).

Al ser la educación un fenómeno muy complejo que involucra una serie de elementos, factores, requisitos, condiciones y que, asimismo, está en permanente cambio, evolución o mutación, se requiere de otros modelos pedagógicos que sintonicen con las exigencias de la realidad actual. En consecuencia, "La formación, tanto en el área académica como pedagógica, por parte de los docentes de educación superior es necesaria para permitir el acceso a la participación y al aprendizaje a todos los alumnos" (Rodríguez Ugalde, Mendoza Saucedo \& Méndez Pineda, 2018, p. 176).

Bajo las perspectivas actuales de la educación superior, la universidad tiene nuevos retos prioritarios relacionados no solo con la forma de construir el conocimiento sino con la apropiación del mismo. 


\section{Referencias bibliográficas}

Almonacid-Fierro, A., Merellano-Navarro, E. \& Moreno-Doña, A. (2014). Caracterización del saber pedagógico: Estudio en profesorado novel. Revista Electrónica Educare, 18(3), 173-190. Recuperado de: http://www.scielo.sa.cr/pdf/ree/v18n3/a10v18n3.pdf

Antón-Candanedo, A. \& Fernández-Río, J. (2017). Hibridando modelos pedagógicos para la mejora de la comprensión táctica de estudiantes: una investigación a través del Duni. Ágora para la educación física y el deporte, 19(2-3), 257-276. Ediciones Universidad Valladolid. Recuperado de: https://dialnet.unirioja.es/descarga/articulo/6261763.pdf

Barajas, G. A. (2013). El acto pedagógico y el modelo pedagógico institucional. Recuperado de: https://dialnet.unirioja.es/descarga/articulo/4966232

Carrasco, C., Pérez, C., Torres, G. \& Fasce, E. (2016). Relación entre prácticas pedagógicas y estrategias de aprendizaje en docentes de carreras de la salud. Rev. Med. Chile(144), 11991206. Recuperado de: https://scielo.conicyt.cl/pdf/rmc/v144n9/art15.pdf

Cartuche, N., Tusa, M., Aguinsaca, J., Merino, W. \& Tene, W. (2015). El modelo pedagógico en la práctica docente de las universidades públicas del país. En M. E. Ortiz-Espinoza, E. Fabara-Garzón, E. Isch-López, \& C. M. Crespo-Burgos (Eds.), Reflexiones sobre la formación y el trabajo docente en Ecuador y América Latina (1. ${ }^{a}$ Edición, 203-231). Quito, Ecuador: Universidad Politécnica Salesiana.

Castillo-Cedeño, I., Flores-Davis, L. E., Jiménez-Corrales, R. E., \& Perearnau-Torras, M. Á. (2008). Una reflexión necesaria. Posibilidad de la construcción de un modelo pedagógico en la educación superior. Revista Electrónica Educare, XII(1), 123-134.

Claret, A. (2003). Las teorías pedagógicas, los modelos pedagógicos, los modelos disciplinares y los modelos didácticos en la enseñanza y aprendizaje de las ciencias. En A. Claret (Ed.), Educación y formación del pensamiento científico, 21-45. Bogotá, Colombia: Arfo Editores e Impresores Ltda.

De Jesús, M. I., Méndez, R., Andrade, R. \& Martínez, D. R. (2007). Didáctica: docencia y método. Una visión comparada entre la universidad tradicional y la multiversidad compleja. Revista de teoría y didáctica de las ciencias sociales(12), 9-29. Recuperado de: http://www. redalyc.org/articulo.oa?id $=65201201$

Díaz-Quero, V. (2006). Formación docente, práctica pedagógica y saber pedagógico. Laurus, 12(Ext), 88-103. Recuperado de: http://www.redalyc.org/articulo.oa?id=76109906

Flórez-Ochoa, R. (2005). Docente del siglo XXI. Cómo desarrollar una práctica docente competitiva. Evaluación, pedagogía y cognición. Bogotá: McGraw Hill.

Flórez-Ochoa, R. (1994). Hacia una pedagogía del conocimiento. Santafé de Bogotá D.C.: McGraw Hill.

Flórez-Ochoa, R. (1999). Evaluación pedagógica y cognición. Santafé de Bogotá D.C.: McGraw Hill.

Fundación Universitaria Luis Amigó. (2009). Identificación del Modelo Pedagógico y construcción de una propuesta de acompañamiento para la formulación o revisión y ajuste de un enfoque-modelo pedagógico. Recuperado el 21 de febrero de 2017, de https://www. encuestafacil.com/RespWeb/Cuestionarios.aspx?EID=1714328\&MSJ=NO\#Inicio 
García Jiménez, M. C. (2009). Corrientes críticas a la escuela tradicional. Innovación y experiencias educativas(14), 1-9. Recuperado de: https://archivos.csif.es/archivos/andalucia/ ensenanza/revistas/csicsif/revista/pdf/Numero_14/M_CARMEN_GARCIA_2.pdf

González Guerrero, K. \& Esteban Ojeda, C. (2013). Caracterización de modelos pedagógicos en formación e-learning. Revista Virtual Universidad Católica del Norte(39), 4-16. Recuperado de: http://www.redalyc.org/articulo.oa?id=194227509002

Hernández, R., Fernández, C. \& Baptista, M. (2016). Metodología de la investigación (6. a ed.). México D.F.: McGraw Hill.

Mantilla-Falcón, L. M., Galarza-Galarza, J. C. \& Zamora-Sánchez, R. A. (2017). La inserción de la mujer en la educación superior ecuatoriana. Caso Universidad Técnica de Ambato. Revista Latinoamericana de Estudios Educativos, 13(2), 12-29. Recuperado de: http://www. redalyc.org/pdf/1341/134154501002.pdf

Morales-Gómez, G. (2004). El giro cualitativo de la educación (10. a ed.). Bogotá: s.e.

Noguera-Ramírez, C. E. \& Marín-Díaz, D. L. (2017). Saberes, normas y sujetos: cuestiones sobre la práctica pedagógica. Educar em Revista(66), 37-56. Recuperado de: http://www. scielo.br/pdf/er/n66/0104-4060-er-66-37.pdf

Pineda-Báez, C., Hennig, C. \& Segovia, Y. (2013). Modelos pedagógicos, trabajo colaborativo e interacción en programas virtuales de pregrado en Colombia: Un camino por recorrer. RUSC. Universities and Knowledge Society Journal, 10(2), 187-202. Recuperado de: http://www. redalyc.org/articulo.oa?id $=78028681014$

Pinilla, A. E. (2011). Modelos pedagógicos y formación de profesionales en el área de la salud. Acta Médica Colombiana, 36(4), 204-218.

Pinto-Blanco, A. M. \& Castro-Quitora, L. (2008). Los modelos pedagógicos. Universidad Abierta(7). Recuperado de: https://mefistocastellano.files.wordpress.com/2015/09/pintoa-castro-I-los-modelos-pedagogicos.pdf

Quero Virla, M. (2010). Confiabilidad y coeficiente Alpha de Cronbach. Telos, 12(2), 248-252. Recuperado de: https://www.redalyc.org/pdf/993/99315569010.pdf

Restrepo-García, R., Franco-Muñoz, N. E. \& Quiroz-Posada, R. E. (2011). Educación superior e imaginarios de género. UNI-PLURIIVERSIDAD, 11(1), 1-8.

Rodríguez Ugalde, D. C., Mendoza Saucedo, F. \& Méndez Pineda, J. M. (2018). Diagnóstico sobre las buenas prácticas docentes en la Facultad de Contaduría y Administración y la Facultad de Derecho de la Universidad Autónoma de San Luis Potosí. Revista Latinoamericana de Educación Inclusiva, 12(1), 163-179. Recuperado de: https://scielo.conicyt.cl/pdf/rlei/ v12n1/0718-7378-rlei-12-01-00163.pdf

Soto Arango, D. E., Mora García, J. P. \& Lima Jardilino, J. R. (2017). Formación de docentes y modelo pedagógico en la Universidad Pedagógica y Tecnológica de Colombia. Rev. hist.edu. latinoam., 19(29), 35-66. Recuperado de: http://www.scielo.org.co/pdf/rhel/v19n29/01227238-rhel-19-29-00035.pdf

Vaccarezza Garrido, G., Sánchez Soto, I. \& Alvarado Martínez, H. (2018). Caracterización de prácticas pedagógicas en carreras de ingeniería civil de universidades de Chile. Espacios, 39(15), 1-16. 


\section{Nota:}

\section{Contribución de autoría}

La concepción y construcción del estado del arte del trabajo científico fueron realizadas por Luis Marcelo MantillaFalcón. La recolección de datos estuvo a cargo de Carlos Fernando Meléndez Tamayo. La revisión del manuscrito fue realizada por Darwin Patricio Miranda Ramos y Grace Elaine Ortega Zurita. Los cuatro autores revisaron y aprobaron el contenido final.

\section{$*, * *, * * * y * * * *$ Universidad Técnica de Ambato (Ecuador).}

${ }^{1}$ Doctor en Ciencias de la Educación, Universidad Técnica de Ambato (Ecuador). Magister en Docencia Universitaria y Administración Educativa, Universidad Tecnológica Indoamérica (Ecuador). Magister en Ciencias de la Educación, Pontificia Universidad Católica del Ecuador. Licenciado en Ciencias de la Educación, Universidad Técnica de Ambato (Ecuador) Diploma en Gerencia con PNL, Universidad Estatal de Bolívar (Ecuador). Especialista en TIC para la práctica docente. Docente investigador, Universidad Técnica de Ambato.

2 Doctor en Ciencias de la Educación y Magister en Diseño Curricular y Evaluación Educativa, Universidad Técnica de Ambato (Ecuador). Máster en Ciencias Naturales, Biología, Geología y Química, Universidad Complutense de Madrid (España). Diploma Superior en Investigación de la Educación a Distancia y Diploma Superior en Investigación Científica y Asesoría Académica, Universidad Regional Autónoma de los Andes (Ecuador). Licenciado en Ciencias de la Educación, Universidad Técnica de Ambato.

${ }^{3}$ Doctora en Gerencia Educativa, Magister en Diseño Curricular y Evaluación Educativa, Licenciada en Ciencias de la Educación y Profesora de Educación Primaria, Universidad Técnica de Ambato (Ecuador). Docente, Universidad Técnica de Ambato.

${ }^{4}$ Ph.D. en Educación, Universidad Complutense de Madrid (España). Máster en Gerencia de la Educación Abierta, Especialista en Proyectos de Investigación Científica y Tecnológica, Ingeniero en Sistemas Informáticos, Profesor-Investigador, Universidad Técnica de Ambato (Ecuador). Director, Departamento de Educación continua a distancia y virtual, Universidad Técnica de Ambato. Evaluador de Proyectos de Investigación, Secretaria Nacional de Ciencia y Tecnología de Ecuador. 